\title{
Acoustical correlates of flute performance technique
}

\author{
N. H. Fletcher
}

Department of Physics, University of New England. Armidale, New South Wales 2351, Australia (Received 21 March 1974; revised 1 August 1974)

\begin{abstract}
Measurements of physical parameters of performance technique for a group of experienced flute players are reported. Blowing pressure is found to be consistent among the players and intermediate between previous measurements by Bouhuys [J. Acoust. Soc. Am. 37, 453-456 (1965)] and by Coltman [J. Acoust. Soc. Am. 40, 99-107 (1966); 44, 983-992 (1968)]. Jet-length measurements agree with those of Coltman. Blowing pressure and jet length come near to satisfying the expected relationship but with some discrepancy which may be significant. Harmonic analysis of flute tone shows that amplitude variations from piano to forte are largely confined to the upper partials. particularly for notes in the low octave. A study of vibrato shows that this normally consists of an amplitude modulation of the upper partials of the tone with little change in the fundamental. The vibrato frequency is consistently about $5 \mathrm{~Hz}$ and is associated with a $10 \%$ variation in blowing pressure at that frequency. Physiological vibrato mechanisms are discussed and the acoustical nature of the vibrato is shown to be determined by the nonlinear jet excitation mechanism and the stabilizing effect of the narrow fundamental pipe resonance.
\end{abstract}

Subject Clasification: 75.35 .

\section{INTRODUCTION}

The particular techniques used by a performer to elicit musical sounds from an instrument are governed both by the acoustical nature of the instrument and by the performer's artistic criteria. Sounds can, indeed, be produced in other ways but will generally be judged to be musically unsatisfying.

During the course of a study with a pedagogic end in view, ${ }^{1}$ a considerable amount of data was collected on flute performance technique by means of measurements made on four experienced players. Two of these (C. E. and L.V.), to be denoted by $A$ and $B$, were experienced professional players, the third (N.F.), denoted by $C$, was an experienced semiprofessional, and the fourth, D, an advanced student (D.M.). To indicate the range of the sample, $\mathrm{A}$ and $\mathrm{C}$ were men and $\mathrm{B}$ and $\mathrm{D}$ were women; $A$ and $B$ were trained predominantly in Australia, while $C$ and $D$ studied both in the United States and in Australia. Measurements should not, the refore, show obvious bias towards a particular school of playing.

The purpose of the present note is to present the results of the measurements, and the conclusions to be drawn from them, in some quantitative detail, and to discuss their relationship to established acoustical principles.

\section{BLOWING PRESSURE}

The correct lip configuration and blowing pressure to be used in playing the flute have been matters of conflicting advice for at least 200 years, ${ }^{2}$ and only recently have careful measurements been made. Bouhuys ${ }^{3}$ has reported measurements of blowing pressure for a variety of wind instruments, including the flute, and Colt$\operatorname{man}^{4}$ has given the results of a rather more extensive series for the flute. Each set, however, involved measurements on one player only and, since the reported pressures differ by almost an order of magnitude, they leave the situation uncertain.

In the present study the air pressure in the player's mouth was measured using a 1-mm catheter tube inserted into one corner of the lip opening. The tube led to a sensitive ane roid pressure gauge which had been calibrated by comparison with a water column. The measurements made on the four players are detailed in Fig. 1. There was some variation in the measurements for the lowest few notes but otherwise the players used consistent pressures for given notes on different occasions. Also shown for comparison are the results of Coltman.

From Fig. 1 it is clear that the blowing pressure used increases roughly linearly with frequency and that there is a good measure of agreement between the four players A, B, C, and D. The median blowing pressure $p$ (in millibars), in excess of atmospheric pressure, to sound a note of frequency $\nu$ (in hertz) is

$$
p \simeq 0.008 \nu,
$$

the maximum observed deviation being mostly $\pm 50 \%$. Measurements on player $\mathrm{C}$ show that this relation also applies for performance on the alto $(G)$ flute.

The results reported by Coltman lie just on the lower edge of this range, but those of Bouhuys (7.5-13 mbar for $C_{4}$ and 27-40 mbar for $A_{6}$ ) are so much higher that they lead to questions. Bouhuys's measurement technique involved insertion of a small latex balloon in the player's mouth, which may have upset the technique or given misleading results. Experiments by the present author suggest that it is simply not possible to produce the lowest notes on the flute with pressures as high as those reported.

Returning to the results shown in Fig. 1 we observe that players $\mathrm{B}$ and $\mathrm{C}$ used substantially the same blowing pressure for a given note for dynamic levels from pianissimo to fortissimo, while $A$ and $D$ varied their blowing pressure by about a factor of 2 over this range. Subjectively, players $B$ and $C$ appeared to mainta in a bright "ringing" tone quality for quiet playing while A and D used a much "softer" tone for quiet passages. 


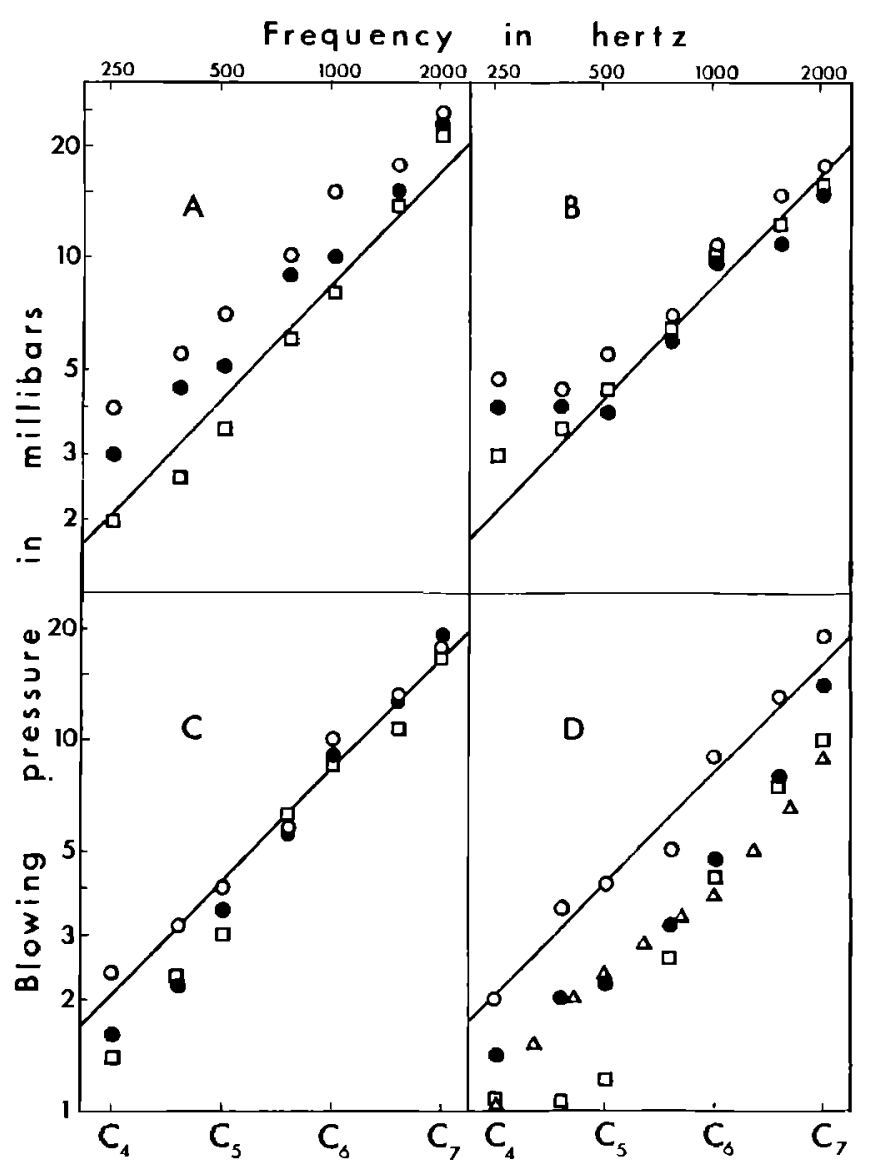

FIG. 1. Blowing pressures $p$ (in millibars) used by flute players $\mathrm{A}, \mathrm{B}, \mathrm{C}$, and $\mathrm{D}$ to produce the notes shown: $\supset$ fortis simo, - mezzoforte, a pianissimo. Measurements by Coltman are also shown: $\Delta$ (mezzoforte). The curve drawn is the relation $p=0.008 \nu$.

This distinction is brought out in the spectral analyses to be discussed later.

\section{LIP CONFIGURATION}

The principal variables to be controlled in the lip configuration, or embouchure, are the width and height of the aperture in the lips producing the air jet, the distance between this aperture and the edge of the flute embouchure hole (the jet length), the fraction of the flute embouchure hole covered by the lower lip, and the angle at which the air jet strikes the flute embouchure edge.

Some of these variables were studied, for each of the four players, by taking photographs, both full face and in profile, and subsequently measuring these. The same technique was used by Coltman ${ }^{4}$ in a similar, though more limited, series of measurements.

Jet length, defined to be the distance measured on a profile photograph from the apparent point of emergence of the jet from the player's lips to the target edge of the flute embouchure :ole, is perhaps the most important and consistent variable. There is a slight arbitrariness about definition of the point of emergence from the lips, but, as we see later, this is not important.

The results of measurements of the four players are shown in Fig. 2. Once more there is good consistency between all four sets of measurements. The median relation between jet length $l$ (in millimeters) and note frequency $\nu$ (in hertz) is

$$
l \simeq 1.8+100 \nu^{-1 / 2}
$$

and all measurements lie within about $\pm 20 \%$ of this relation, the players making little or no adjustment to $l$ with dynamic level of their playing. The analytical form assumed for Eq. 2 is derived from subsequent discussion. The precision of the measurements and range of frequency involved is certainly not sufficient to distinguish between Eq. 2 and a simple linear relation between $l$ and $\nu$.

Coltman ${ }^{4}$ has not reported measurements of jet length directly, but instead has plotted the width of the flute embouchure hole left uncovered by the lower lip. This ranges from $6 \mathrm{~mm}$ for $C_{4}$ to $3.5 \mathrm{~mm}$ for $G_{6}$ and, since it is typically 1-2 mm less than the jet length, his measurements are in substantial agreement with those reported above.

Analysis of the full-face photographs of the four players in this study again shows a good measure of consistency among them in the matter of embouchure shape. The lip opening approximates an oval with an axial ratio between 10:1 and 20:1, although its shape is slightly irregular and varies from player to player. Its maximum width is about $12 \mathrm{~mm}$, equal to the width of the flute embouchure hole, for low notes played loudly, and its size is reduced to produce soft sounds or to play high notes. For very high notes played softly, the embouchure width decreases to about $5 \mathrm{~mm}$, its axial ratio remaining between 10:1 and 20:1.

\section{RELATION TO THEORY}

All these observations are in general agreement with what we should expect from acoustical principles. Colt$\operatorname{man}^{4}$ has made careful measurements of the impedance of an air jet interacting with a flute, after the fashion of

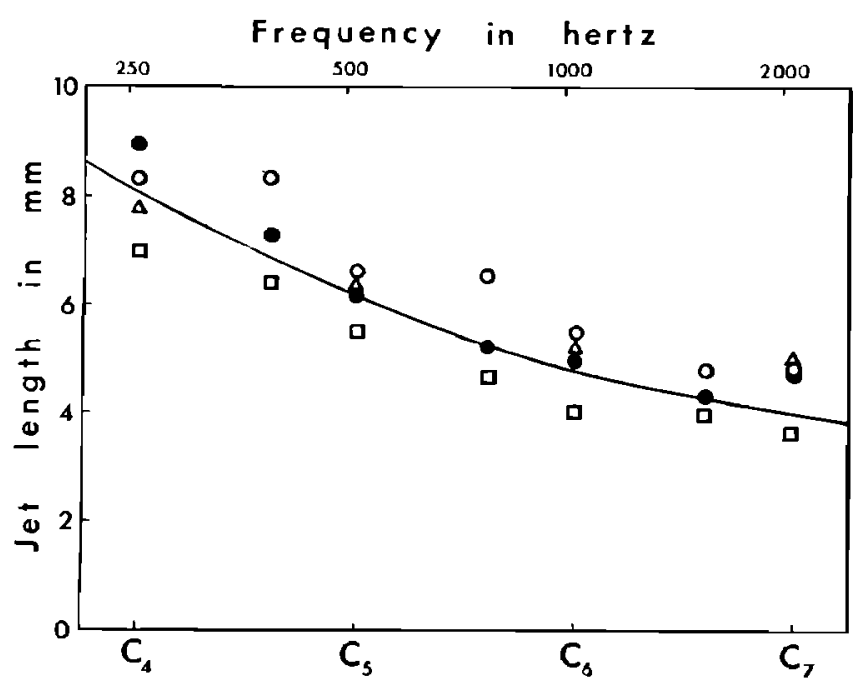

FIG. 2. Air jet lengths $l$ (in millimeters) measured from photographs for the players $A, O ; B, \bullet ; C, \square ; D, \Delta$. The curve drawn is the relation $l=1.8+100 \nu^{-1 / 2}$. 
a normal player, and has identified regions where the jet impedance is negative, leading to sustained oscillation. The pure negative resistance region corresponds to a particle travel time along the length of the jet of about 0.2 of an oscillation period. The apparent fact that the displacement wave velocity along the jet is about 0.4 of the particle velocity, together with an additional phase shift of $\pi$, gives the correct phase relationship for regeneration.

If we assume that jet velocity is determined by Bernoulli's equation and require that the travel time be 0.2 of an oscillation period, then we are led to the relation

$$
l p^{-1 / 2}=2700 \nu^{-1}
$$

where $l$ is in millimeters, $p$ in millibars, and $\nu$ in hertz. More realistically, however, we must allow for the fact that the jet velocity decreases with its travel distance, due to entrainment of stationary air, so that we should replace Eq. 3 by

$$
l p^{-1 / 2}=2700 \alpha \nu^{-1},
$$

where $\alpha<1$. To compare this with our measurements, we see that a simple manipulation of Eqs. 1 and 2 gives the relation

$$
(l-1.8) p^{-1 / 2}=1100 \nu^{-1} .
$$

The two results, Eqs. 4 and 5, can be reconciled if we recognize that the measured length $l$ contains a dead interval of $1.8 \mathrm{~mm}$ which is effectively shielded from the acoustic field and assume that the factor $\alpha$ has a value near 0.4 so that the average jet speed is a little less than half that predicted from Bernoulli's equation.

There are, however, other possibilities. Coltman's measured blowing pressures ${ }^{4}$ are only half those found in the present study for nearly the same jet length, which would increase the right-hand side of $\mathrm{Eq}$. 5 by a factor 1.4, leading to closer agreement. There is also a distinct feeling among flute players that, rather than blowing the "center" of a note, which might correspond to the optimum phase condition Eq. 4, one actually blows just short of the condition which would give overblowing to the next octave. In this way the harmonic development of the tone is enriched and sensitive adjustment of embouchure hole coverage prevents increase in frequency. Coltman's experiments ${ }^{4}$ with an artificially blown flute suggest that this increase in frequency should be a sensitive test of blowing condition, but the present author's experiments with organ pipes (to be reported later) show a stable frequency regime for an energetically blown pipe. The details of this point therefore remain to be resolved.

Another point of disagreement concerns the way in which increases of dynamic level from piano to forte are achieved. Our measurements show that, while some players may make adjustments of blowing pressure, there is a universal adjustment of the lips to increase the cross section of the jet when a forte level is required. Coltman, on the other hand, found that, with his artificially blown flute, "radiated power turned out to be a function mostly of blowing pressure, and was not very dependent on the note being produced or the size of the blowing tube." The subtle factor involved here may well be the shape of the cross section of the blowing jet, since calculations ${ }^{6}$ have shown this to be of considerable importance in determining the amplitudes of the upper partials, as well as of the fundamental.

\section{HARMONIC DEVELOPMENT}

Many texts on musical acoustics characterize flute tone as being of weak harmonic development and imply that the fundamental is always dominant. The harmonic development of flute tone is, of course, much less rich than that of oboe tone, but in the lowest octave the fundamental is by no means dominant.

To clarify these matters and to seek for possible correlation between harmonic development and performance technique, the sounds produced by each of the four players in this study were recorded and analyzed. A dynamic microphone having a hypercardioid response pattern was used at a distance of about $1 \mathrm{~m}$ from the player in a normal living room. The same room was used for $A$ and $B$ while $C$ and $D$ were in different but rather similar rooms. The recordings were judged subjectively to give a faithful reproduction of the sound as heard, but may obviously contain some emphasis of certain frequencies due to standing waves. They are, however, probably preferable to recordings made in anechoic surroundings because of the effect of such an unusual environment on the player.

Figure 3 shows the results of harmonic analysis of the sounds produced by each of the four players for three different notes, each played both loudly and softly. Again, there is good general agreement among all players, but some differences. The main conclusions are as follows, all partials being strictly harmonic.

In the lowest octave of the flute, and for loud playing, the fundamental is lower in level than either the second or third harmonics, and may be lower than the fourth and fifth harmonics as well. When the playing is soft in this octave, the level of the fundamental is the same as for loud playing but the relative levels of all higher harmonics are decreased. This decrease is much more pronounced for players $A$ and $D$, who reduce wind pressure for soft playing, than for B and C, who use a constant wind pressure.

For the middle octave of the flute, the fundamental becomes the dominant partial for both loud and soft playing, though second and third harmonics are within $10 \mathrm{~dB}$ of it in relative level. The sound-pressure level of the fundamental changes little with dynamic level and most of the change is represented by changes in the upper partials.

In the third octave the fundamental is clearly dominant and all upper partials are more than $10 \mathrm{~dB}$ below it in relative level. The fundamental changes considerably with dynamic level, though still not as much as do the upper partials.

If we were to seek to describe a formant for flute tone on the basis of these measurements, then we would in 


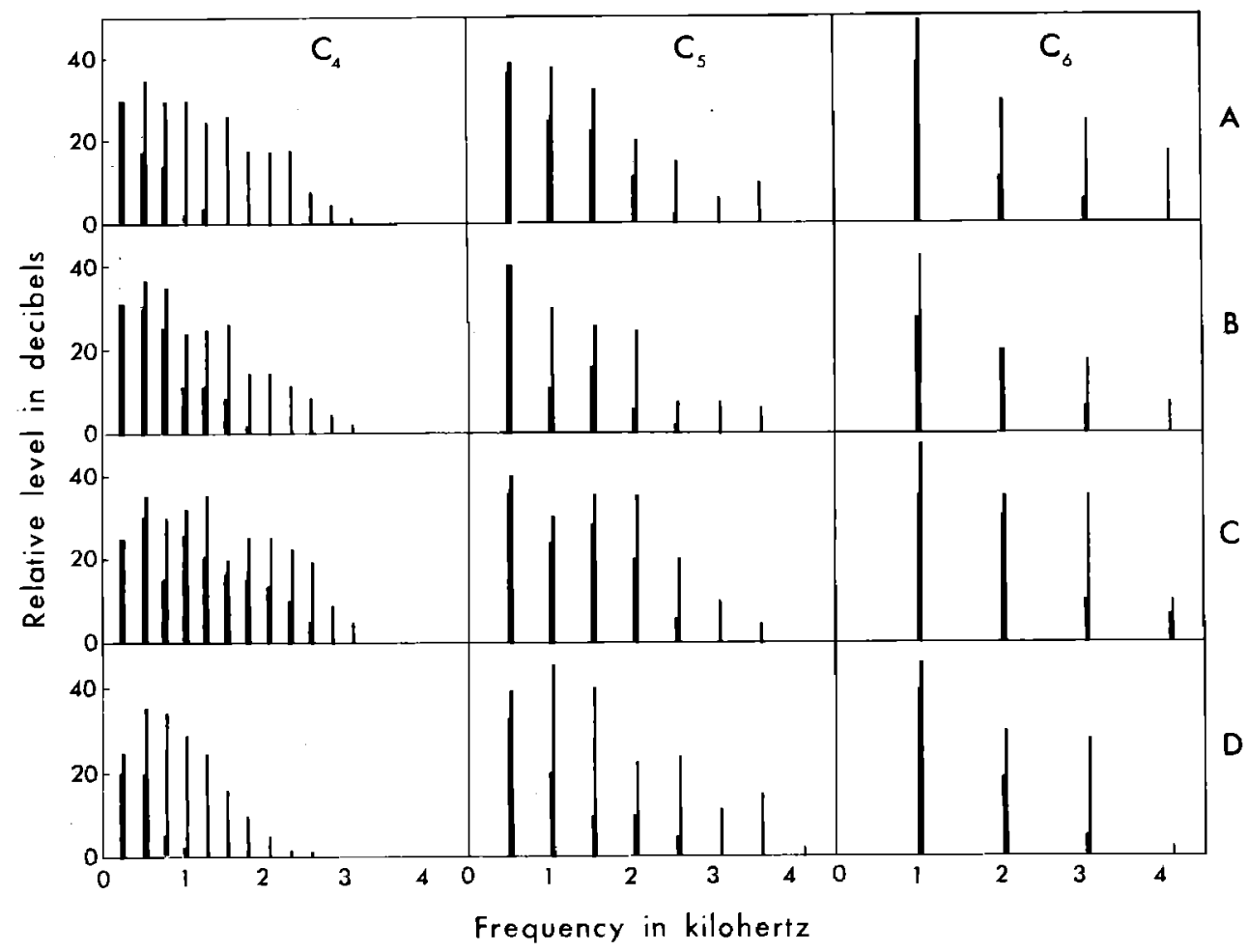

FIG. 3. Harmonic analysis of forte and piano notes played by $A, B, C$, and D. Absolute soundpressure levels were not determined and relative levels of partials are given in decibels consistently for each player separately.

fact need different formants for loud and soft playing. For forte tone the formant rises at about $12 \mathrm{~dB} /$ octave up to $500 \mathrm{~Hz}$, is constant from 500 to $1000 \mathrm{~Hz}$, and above this falls at about $12 \mathrm{~dB}$ /octave. For piano playing the upper roll-off point is reduced to about $500 \mathrm{~Hz}$ and the rising lower portion of the formant curve is either reduced in extent or even eliminated.

The nonlinear mechanism responsible for hamonic generation in flutes and organ pipes is not yet fully understood, but an approximate theory has been developed by the present author ${ }^{5}$ based on some more qualitative arguments by Benade. ${ }^{6}$ To maximize harmonic development it appears desirable to use a very thin lamellar jet to maximize the nonlinearity of its interaction with the embouchure edge. It is also desirable to operate at a wind pressure just short of that giving overblowing to the octave. In this regime, indeed, the level of the fundamental may even decrease with increased blowing pressure, while the levels of most of the upper partials increase. This is in general agreement with the results of our measurements, though any detailed calculation for upper partials must await a further development of the theory.

\section{VIBRATO}

The various components of flute vibrato can be readily dissected from recorded sounds by using a wave analyzer, provided that the bandwidth of the analyzer is much larger than either the amplitude-modulation frequency (the pulsation frequency) or the frequency deviation in the vibrato. For a normal flute vibrato the pulsation frequency is about $5 \mathrm{~Hz}$ and the frequency deviation is less than $1 \%$, so that the measurement conditions for the first few harmonics are met by using a heterodyne wave analyzer (GR 1900-A) with a fixed 50-Hz bandwidth.
The vibrato of subjects $A, B$, and $C$ of the present study was analyzed in this way and found to be very similar. Subject $\mathrm{C}$ was then further analyzed as discussed below.

Analysis of the individual partials of flute tone with vibrato shows frequency modulation of less than $1 \%$, but substantial amplitude modulation which varies from one partial to another. The amplitude variation is only roughly sinusoidal but we can define an approximate modulation index $\delta_{n}$ for the $n$th harmonic by writing its behavior as $a_{n}\left(1+\delta_{n} \sin \Omega t\right) \sin n \omega t$ for its sound pressure. The modulation frequency $\Omega$ is very close to $5 \mathrm{~Hz}$ while the $a_{n}$ (sound-pressure amplitude in unspecified units) and $\delta_{n}$ have the values given in Table I for a low note $\left(C_{4}=262 \mathrm{~Hz}\right.$ nominally) and a midrange note $\left(C_{5}=524 \mathrm{~Hz}\right)$.

From this table it is apparent that flute vibrato consists largely of an amplitude modulation of the upper partials of the tone, causing a periodic variation both in loudness and, more importantly, in timbre. Once this is realized, the timbre vibrato aspect of the tone quality is immediately apparent to the listener.

Nearly all flute players produce vibrato by an oscillation of the diaphragm muscles to produce a rhythmic fluctuation in blowing pressure. Direct measurements by a pressure transducer, linked to the player's mouth cavity by a $1-\mathrm{mm}$ catheter tube, confirm that the pres-

TABLE I. Amplitudes $a_{n}$ and modulation indices $\delta_{n}$ for vibrato.

\begin{tabular}{lrlrlllll}
\hline \hline Note & $a_{1}$ & $\delta_{1}$ & \multicolumn{1}{c}{$a_{2}$} & \multicolumn{1}{c}{$\delta_{2}$} & $a_{3}$ & $\delta_{3}$ & $a_{4}$ & $\delta_{4}$ \\
\hline$C_{4}$ & 4 & 0.1 & 12 & 0.2 & 7 & 0.4 & 6 & 0.7 \\
$C_{5}$ & 11 & 0.2 & 2 & 0.5 & 4 & 0.5 & 4 & 0.5 \\
\hline \hline
\end{tabular}


sure fluctuations have a frequency of $5 \mathrm{~Hz}$ and an amplitude about $10 \%$ of the blowing pressure. A few players are known to use a lip-driven or throat-driven vibrato but this is unusual and is not generally regarded as being very satisfactory.

Experience shows that it is not very easy for the player to vary his vibrato frequency and that this frequency is very nearly the same for all players. This suggests that some sort of resonant mechanism may be involved and there seem to be two possibilities, one mechanical and one neurophysiological.

In the first place we note that the lung cavity contains a volume $V \sim 10^{-2} \mathrm{~m}^{3}$ of air maintained under a pressure $p \sim 10^{5} \mathrm{~Pa}$ slightly above atmospheric by an elastic diaphragm of area $A \sim 3 \times 10^{-2} \mathrm{~m}^{2}$. The diaphragm is loaded by the mass $m \sim 10 \mathrm{~kg}$ of the contents of the abdomen. The resonant frequency of this system is $\nu=(1 / 2 \pi)\left(p A^{2} /\right.$ $m V)^{1 / 2} \simeq 5 \mathrm{~Hz}$, which corresponds closely to the observed vibrato frequency. It is therefore reasonable that one can play with a "straight" tone without vibrato, but that, once vibrato is allowed to develop, it tends to run at the resonant frequency.

The second possibility is that the diaphragm is maintained in the correct state of tension by opposing sets of muscles which are controlled by both voluntary and involuntary neurological feedback loops. The oscillation frequency of such loops, as observed in various nervous disorders, is also not far from $5 \mathrm{~Hz}$. It is most likely, of course, that both mechanisms are involved, but experiments are being planned to try to separate these.

Returning to the acoustical effects of the periodically varying blowing pressure, we find that the effects shown in Table I can readily be accounted for. The data of Fig. 1 show that variations in loudness are achieved through variation in amplitude of the upper partials rather than of the fundamental. Wormann, ${ }^{8}$ in a study of clarinet tone, has shown that, over a limited range, the amplitude of the $n$th harmonic varies as the $n$th power of the amplitude of the fundamental, and calculations by the present author ${ }^{6}$ show that, for a strongly blown pipe, the amplitude of the fundamental remains constant or even decreases with increased blowing pressure, while the amplitude of the second harmonic increases sharply.

In addition to these steady-state effects, the timevarying nature of the vibrato must be taken into account. Using the method described by Benade, ${ }^{7}$ we have measured the acoustic resonances of a flute tube and head joint with simulated lips in position. Because of the narrow tube diameter $(19 \mathrm{~mm})$ and the presence of the correcting cavity and taper in the head joint, ${ }^{9}$ the resonances are in harmonic relation to a very good approximation. For the tube fundamental $C_{4}=262 \mathrm{~Hz}$, the resonance half-widths at $3-\mathrm{dB}$ points are $\Delta_{1}= \pm 4, \Delta_{2}= \pm 6$, $\Delta_{3}= \pm 7, \Delta_{4}= \pm 8 \mathrm{~Hz}$, corresponding to quality factors ( $Q$ values) for these resonances in the range 40-70. The small width of the fundamental resonance will attenuate the side bands at $\pm 5 \mathrm{~Hz}$ carrying amplitude-modulation information and thus smooth the output. Because of their greater widths, the upper resonances will have a smaller smoothing effect. This phenomenon reinforces the effect of the blowing mechanism in favoring modulation of upper partials and, indeed, the reverberant nature of the rooms in which the recordings were made has a similar effect.

\section{ACKNOWLEDGMENTS}

This work is part of a program, aimed at elucidating second-order effects in the design and performance of traditional musical instruments, which is supported by the Australian Research Grants Committee.

${ }^{1}$ N. H. Fletcher, Instrumentalist 28(7), 57-61 (1974).

${ }^{2}$ J. J. Quantz, On Playing the Flute (1752), transl. by E. R. Reilly (Faber and Faber, London, 1966), pp. 49-59.

${ }^{3}$ A. Bouhuys, J. Acoust. Soc. Am. 37, 453-456 (1965).

${ }^{4}$ J. W. Coltman, J. Acoust. Soc. Am. 40, 99-107 (1966).

JJ. W. Coltman, J. Acoust. Soc. Am. 44, 983-92 (1968).

${ }^{6}$ N. H. Fletcher, J. Acoust. Soc. Am. 56, 645-652 (1974).

${ }^{7}$ A. H. Benade, J. Acoust. Soc. Am. 40, 247-249 (1966).

${ }^{8}$ W. Wormann, Ph. D. thesis, Case Western Reserve University, (1970), p. 53.

${ }^{9}$ A. H. Benade and J. W. French, J. Acoust. Soc. Am. 37, 679-691 (1965). 